\title{
Erratum to: The Cusp/Core problem: supernovae feedback versus the baryonic clumps and dynamical friction model
}

\author{
A. Del Popolo ${ }^{1,2,3} \cdot$ F. Pace ${ }^{4}$
}

Published online: 14 June 2016

(C) Springer Science+Business Media Dordrecht 2016

\section{Erratum to: Astrophys Space Sci (2016) 361:162 \\ DOI 10.1007/s10509-016-2742-z}

In the original publication, the first figure (labeled Fig. 1) is actually Fig. 2 with the caption of Fig. 1 and vice versa. The correct figures plus captions are shown below. (What is said about the figures in the text of the original article should be read based on the information as shown below.)
The online version of the original article can be found under doi:10.1007/s10509-016-2742-z.

$\bowtie$ A. Del Popolo

adelpopolo@oact.inaf.it

1 Dipartimento di Fisica e Astronomia, Università di Catania, Viale Andrea Doria 6, 95125 Catania, Italy

2 INFN sezione di Catania, Via S. Sofia 64, 95123 Catania, Italy

3 International Institute of Physics, Universidade Federal do Rio Grande do Norte, 59012-970 Natal, Brazil

4 Jodrell Bank Centre for Astrophysics, School of Physics and Astronomy, The University of Manchester, Manchester, M13 9PL, UK

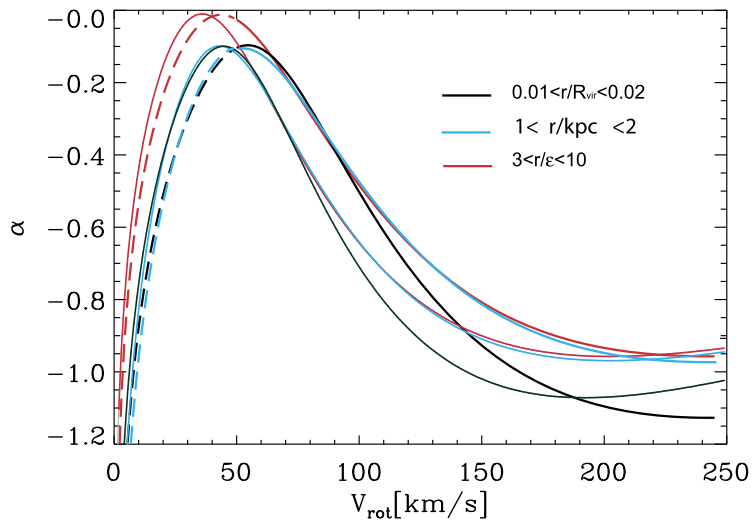

Fig. 1 The $\alpha-V_{\text {rot }}$ relation. The dashed lines refer to the calculations of Di Cintio et al. (2014), and the solid lines are those calculated in this paper. The dashed lines and the solid ones are different by a factor $\mathrm{h}$ inverse, coming from the Tully-Fisher relation of Dutton et al. (2010), and discarded in Di Cintio et al. (2014). The black lines refer to the slope calculated at $0.01<r / R_{\text {vir }}<0.02$, the cyan lines that calculated at $1<r / \mathrm{kpc}<2$ and the red lines refer to the slope calculated at $3<r / \epsilon<10$ 


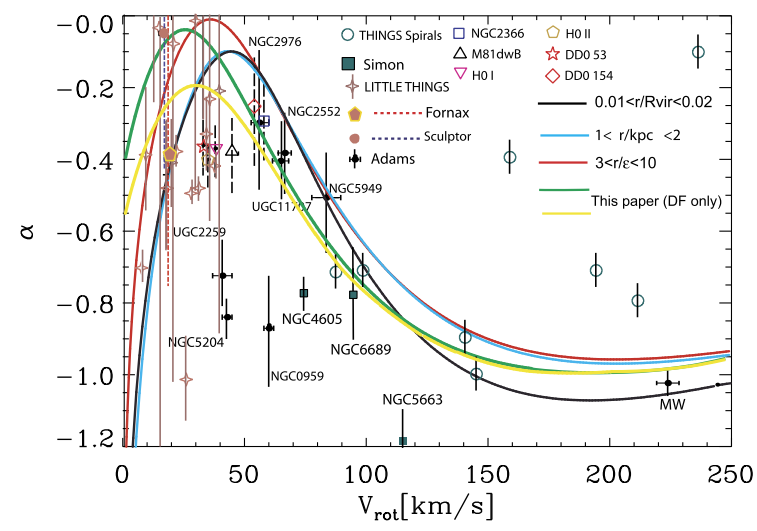

Fig. 2 The $\alpha-V_{\text {rot }}$ relation compared to observations. Data come from Adams et al. (2014), Simon et al. (2005) (excluding the galaxies studied by Adams et al. 2014), the THINGS spirals, the THINGS dwarves, the LITTLE THINGS, Fornax, and Sculptor (Walker and Peñarrubia 2011) and the MW (Ascasibar et al. 2006). The red, black, and blue lines describe the model of Di Cintio et al. (2014), while the green

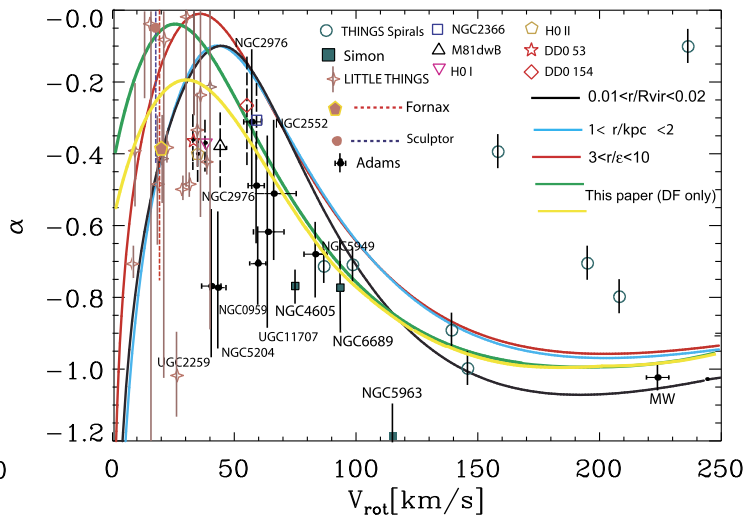

and yellow lines the model of the present paper. The only difference between the left and right panel is due to the galaxy sample in Adams et al. (2014): gas traced, in the left panel; stellar traced, in the right panel. The green (yellow) curves are our equivalent for the red (cyan) curve of Di Cintio et al. (2014) 